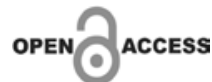

CCESS
RELATO DE CASO

\section{Manifestações Otorrinolaringológicas da Miastenia Gravis: Relato de Caso}

\author{
Nilvano Andrade ${ }^{1}$ \\ ${ }^{1}$ Departamento de Otorrinolaringologia do Hospital Santa Izabel;Salvador, Bahia, Brazil
Otorhinolaryngological Manifestations of Myasthenia Gravis: Case Report

\begin{abstract}
A Miastenia Gravis é uma doença autoimune, neurológica, rara, que afeta o sistema músculo-esquelético. Fadiga e fraquezas musculares, além de sintomas otorrinolaringológicos, como disartria e disfagia, são os mais comuns. Este artigo descreve o relato de um caso clínico de Miastenia Gravis em um idoso de 75 anos. Através de um acompanhamento multidisciplinar (fonoaudiologia, fisioterapia, otorrinolaringologia e neurologia) e utilização da piridostigmina o paciente evoluiu bem sendo encaminhado para seguimento e acompanhamento da doença. É de fundamental importância o diagnóstico precoce frente às queixas otorrinolaringológicas para o manejo adequado da Miastenia Gravis. Palavras-chave: Miastenia Gravis; Suspeita; Tratamento.
\end{abstract}

Correspondence addresses:

Dr. Nilvano Andrade

nilvano@gmail.com

Received: December 13, 2020

Revised: January 7, 2021

Accepted: February 19, 2021

Published: March 31, 2021

Data Availability Statement: All relevant data are within the paper and its Supporting Information files.

Funding: This work was the result of author's initiative. There was no support of research or publication funds.

Competing interests: The author has declared that no competing interests exist.

\section{Copyright}

(C) 2021 by Santa Casa

de Misericórdia da Bahia.

All rights reserved.

ISSN: 2526-5563

DOI 10.35753/rchsi.v5i1.196
Myasthenia Gravis is a rare autoimmune and neurological disease that affects the musculoskeletalsystem. Musclefatigueandweakness, as wellasotorhinolaryngological symptoms, such as dysarthria and dysphagia, are the most common complaints. This article describes a case report of Myasthenia Gravis in a 75-year old man. Through multidisciplinary monitoring (phonoaudiology, physiotherapy, otorhinolaryngology, and neurology) and the use of pyridostigmine, the patient progressed well and was referred for follow-up and monitoring of the disease. Early diagnosis in the face of otorhinolaryngological complaints is of fundamental importance for the proper management of Myasthenia Gravis.

Keywords: Myasthenia Gravis; Suspicion; Treatment.

\section{Introdução}

A Miastenia Gravis é uma doença neurológica crônica, autoimune, que afeta a porção pós-sináptica da junção neuromuscular. Trata-se de uma patologia de incidência rara e muitas vezes subdiagnosticada. Nas últimas décadas, a Miastenia Gravis vem apresentando aumento em sua curva de crescimento, que passou a uma incidência de 9 a 21 por milhão de habitantes com uma prevalência de 150 a 250 casos por milhão de habitantes. ${ }^{1-3}$ Manifesta-se usualmente em adultos jovens ou em idosos, com predomínio do sexo feminino na terceira e quarta décadas de vida. ${ }^{1-4}$

A principal característica da doença é a sensação de fraqueza e fadiga dos músculos esqueléticos de uso repetitivo. Os sintomas otorrinolaringológicos 
correspondem a 15\% dos sintomas iniciais, evolvendo na maioria das vezes de modo assimétrico: fraqueza bulbar, disartria $\mathrm{e}$ disfagia. ${ }^{1,2}$

Há décadas a doença apresentava uma alta taxa de mortalidade, resultando no termo Miastenia Gravis. Os avanços no diagnóstico e tratamento alvo proporcionaram um decréscimo importante nas taxas de mortalidade atual, com relatos em literatura que apontam 0,06 a 0,89 de mortes por milhão/pessoas/ano. ${ }^{4}$ Este artigo tem como objetivo apresentar as principais manifestações otorrinolaringológicas associadas à Miastenia Gravis apresentadas por um paciente admitido no serviço de urgência de Otorrinolaringologia do Hospital Santa Izabel.

\section{Relato de Caso}

NSIM, sexo masculino, 75 anos, admitido no setor de urgência otorrinolaringológica do Hospital Santa Izabel, Salvador, Bahia. O paciente recebeu o primeiro atendimento pela equipe médica com queixa de disfonia há 3 meses acompanhada de disfagia progressiva há 1 mês. Ele relatava piora importante há 4 dias, associado a ptose palpebral bilateral e dispneia nas últimas 24 horas.

Figura 1. Exame antes da admissão.

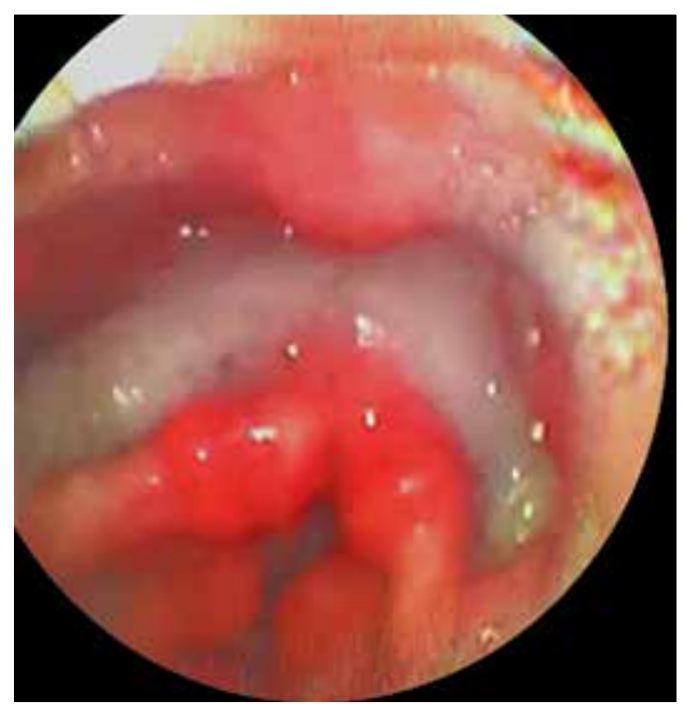

Ao exame físico, destacava-se uma limitação demobilidade dalíngua. Ànasofibrolaringoscopia apresentava insuficiência velofaringea e estase salivar com clearance lentificado, sem evidências de lesões faríngeas e laríngeas. A tomografia de crânio sem método de contraste realizada nas primeiras horas da admissão afastou eventos agudos ou lesões do parênquima cerebral. A realização de exame de imagem de controle, após 8 horas da sua admissão, afastou evento isquêmico. Após descartada possibilidade de evento central, aventou-se a suspeita de doença da placa motora, sendo encaminhado à equipe de neurologia clínica.

O paciente foi transferido para unidade de terapia intensiva, devido à iminência de obstrução de via áerea, onde recebeu acompanhamento multidisciplinar (fonoaudiologia, fisioterapia, otorrinolaringologia e neurologia). Foi mantido em ventilação espontânea e em uso de sonda nasoenteral e medidas de suporte.

Diante do quadro clínico compatível de doença miastênica, foi optado por iniciar teste terapêutico com pirostigmina. Após 48 horas de início do tratamento, foi observada melhora significativa do quadro clínico. A nasofibroscopia de controle evidenciou a redução da estase salivar além do retorno da patência laringofaríngea (Figuras 1 e 2).

Figura 2. Exame pós-tratamento.

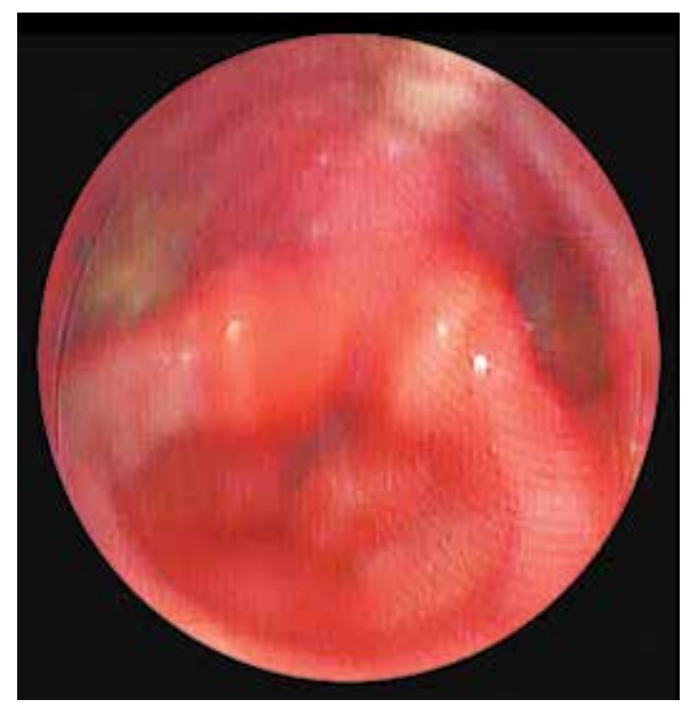




\section{Discussão}

Apesar da sua baixa incidência, a Miastenia Gravis é a principal doenças que afeta a junção neuromuscular.

$\mathrm{Na}$ maioria dos pacientes não se identificam fatores precipitantes no início da doença. Porém, já foi relato, minoritariamente, uma relação com doenças infecciosas virais como: herpes simples, hepatite $\mathrm{C}$ e outras doenças infecciosas. Existem ainda relatos de associação causal com estresse emocional, cirurgias, traumas, uso de antibióticos e gestação, sendo a predisposição genética fator preponderante para o desenvolvimento da doença. ${ }^{4,5}$

Uma característica marcante no distúrbio é seu grau flutuante fruto de um ataque imunológico, mediado por células $\mathrm{T}$ e dirigido a proteínas da membrana pós-sináptica na junção neuromuscular (receptores de acetilcolina e/ou proteínas associadas a receptores) ${ }^{4}$

$\mathrm{Na}$ maioria das vezes, os pacientes experimentarão pelo menos uma exacerbação dos sintomas durante o curso da doença, aumentando o risco de complicações. Há, principalmente, o predomínio de distúrbios da motricidade dos músculos oculares, faciais, bulbares e das extremidades, com um menor comprometimento dos reflexos, trofismo e tônus. ${ }^{5}$

Agravidade dessa doença pode ser classificada na forma funcional e regional utilizando-se a escada de Osserman, composta de quatro graus:

\section{Doença focal;}

II. Doença generalizada;

III. Doença grave;

IV. Doença com necessidade de ventilação assistida. ${ }^{5,6}$

Tendo em vista a diversidade de manifestações, encontramos na área da Otorrinolaringologia diversas apresentações da Miastenia Gravis. Dentro das manifestações faringolaríngeas, a disartria foi o sintoma primário mais frequente, seguido por disfagia, fala arrastada e disfonia. ${ }^{1,2}$
Graças à integração fisiológica simultânea gerada por estímulos nervosos dos pares cranianos (trigêmio, facial, glossofaríngeo, hipoglosso e vago), diversas estruturas relacionam-se no processo da vocalização e deglutição. Estas devem ser analisadas como sítios de comprometimento da condução nervosa nas junções neuromusculares. ${ }^{7,8}$

Consequentemente, um distúrbio na condução nervosa aplicada a essas estruturas comprometem significativamente o comportamento fisiológico dos sistemas envolvidos, alterando o fechamento glótico, ocasionando presença de resíduos e estase salivar em valéculas e hipofaringe, mudanças na qualidade vocal, paralisia das pregas vocais e/ou a distúrbios da deglutição.

Devido ao amplo espectro de possíveis manifestações clínicas, faz-se necessário estratificar e diferenciar os sintomas que possam mimetizar a Miastenia Gravis.

$\mathrm{Na}$ última década, estudos apontam que as estatísticas de mortalidade para Miastenia Gravis caíram dramaticamente ao longo do tempo. Entre 1940 e 1957, a taxa de mortalidade era de $31 \%$, enquanto entre 1966 e 1985 , a taxa de mortalidade foi de $7 \%$. As duas principais razões para esta taxa de mortalidade reduzida são a melhoria dos cuidados multidisciplinares e intensivos e a introdução do tratamento precoce. ${ }^{4}$

Dentre os principais exemplos a serem considerados nos diagnósticos diferenciais temos: a síndrome miastênica de EatonLambert, o botulismo, a esclerose lateral amiotrófica, a sarcoidose e o tratamento com penicilamina, que pode originar quadro neurológico semelhante. ${ }^{4,8,9}$

O diagnóstico da Miastenia Gravis frente à suspeita clínica, pode ser estabelecido por testes clínicos e sorológicos. O tratamento depende da gravidade da sintomatologia, podendo ser dividido em tratamento sintomático, tratamento modificador de doença e intervenção terapêutica aguda.

No tratamento sintomático, utilizam-se os inibidores da acetilcolinesterase, como 
a piridostigmina, com o intuito de facilitar a transmissão neuromuscular. ${ }^{10}$ Em relação ao tratamento modificador de doença, os glicocorticoides e a azatioprina são os imunossupressores de primeira linha. O tratamento da crise miastênica em situação aguda é realizado através de intervenções que reduzem os anticorpos circulantes como a plasmaférese, administração de imunoglobulina intravenosa ou com pulsos de metilprednisolona. ${ }^{10,11}$

Devido ao impacto negativo desta doença na qualidade de vida do paciente é de fundamental importância a sua suspeita durante investigação clínica em pacientes com sinais e sintomas otorrinolaringológicos.

\section{Conclusão}

Ao longo dos anos, a fisiopatologia da Miastenia Gravis foi gradualmente sendo esclarecida e várias terapias foram desenvolvidas na tentativa de aliviar as manifestações clínicas e retardar a evolução da doença.

$\mathrm{O}$ diagnóstico precoce frente às queixas otorrinolaringológicas é imprescindível para o manejo adequado da Miastenia Gravis, evitando complicações inerentes à evolução natural da doença, diminuindo o acometimento sistêmico que tem elevadas taxas de complicações.

\section{Referências}

1. Yang XL, Niu L, Yang CW, Wang L, Liu JX, He Y. Clinical features of laryngeal myasthenia gravis: A case series. American Journal of Otolaryngology-Head and Neck Medicine and Surgery. 9 October 2018.

2. Gilhus NE. Myasthenia Gravis. N Engl J Med 2016;375:2570-81. DOI: 10.1056/NEJMra1602678.

3. Balabbigari N et al. Cranial nerve IX and X weakness: An unusual initial presentation of Myasthenia Gravis. Am J Case Rep. 2020;21:e923750.

4. Hehir MK, Silvestri NJ. Generalized Myasthenia Gravis classification, clinical presentation, natural history, and epidemiology. Neurol Clin 2018;36:253260. 0733-8619/18/a 2018 Elsevier Inc.

5. Lang B, Vincent A. Autoimmune disorders of the neuromuscular junction. Curr Opin Pharmacol. 2009 Jun;9(3):336-40.

6. Juel VC, Massey JM. Myasthenia gravis. Orphanet J Rare Dis. 2007 Nov 6;2:44.

7. Carr AS, Cardwell CR, McCarron PO, McConville J. A systematic review of population based epidemiological studies in Myasthenia Gravis. BMC Neurol. 2010 Jun 18;10:46.

8. Phillips LH. The epidemiology of myasthenia gravis. Semin Neurol. 2004 Mar;24(1):17- 20.

9. Thanvi BR, Lo TC. Update on myasthenia gravis. Postgrad Med J. 2004 Dec;80(950):690-700.

10. Meriggioli MN, Sanders DB. Autoimmune myasthenia gravis: emerging clinical and biological heterogeneity. Lancet Neurol. 2009;8(5):475-90.

11. Amato A, Russell JA. Disorders of neuromuscular transmission. In: Amato AA, Russell JA. Neuromuscular disorders. New York: McGraw Hill; 2008. p. 457-528. 